\title{
Influence of interfacial roughness on the tunnel magnetoresistance
}

\author{
Yun $\mathrm{Li}^{\mathrm{a}, \mathrm{b}, *}$, Ching-Ray Chang ${ }^{\mathrm{a}}$ \\ a Department of Physics, National Taiwan University, Taipei 106, Taiwan \\ ${ }^{\mathrm{b}}$ Institute of Solid State Physics, Academia Sinica, P.O. Box 1129, 230031 Hefei, PR China
}

Received 9 January 2001; accepted 25 July 2001

Communicated by J. Flouquet

\begin{abstract}
The influence of interfacial roughness on tunnel conductance (TC) and magnetoresistance (TMR) are investigated taking into account spin-orbit (SO) coupling in ferromagnet (FM)/insulator (semiconductor) (I(S))/FM junctions. Explicit expressions for spin relaxation are obtained for ballistic and diffusive transport, respectively. The TC is expressed as the sum of different spin channels. Spin-flip (SF) scattering due to SO coupling is much stronger for antiparallel alignment of magnetization in the two FMs. The SF scattering cause a significant decrease of TMR ratio. We find that surface spin scattering predominates which suggests that the interfacial states play a key role in the tunnel process. The feature of the reduction of TMR changes with the disorder configuration and the SO coupling strength. Resonant TMR occurs due to the hopping between the SO-split energy levels. ๑ 2001 Elsevier Science B.V. All rights reserved.
\end{abstract}

PACS: 73.40.Gk; 73.50.Jt; 75.70.Cn

Keywords: Interfacial roughness; Tunnel junction; Spin-orbit coupling; Spin-flip effect; Tunnel conductance and magnetoresistance

Spin-polarized tunneling has become a very active area of research since the discovery of large tunnel magnetoresistance (TMR) at room temperature in magnetic tunnel junctions (MTJs) [1-7]. The conservation of electron spin during tunneling process is essential to the existence of TMR, therefore spinconserving is assumed throughout the process in most of the theoretical treatments, but actual MTJs contain large amounts of disorder at the interfaces between the FMs and the barrier in the fabrication process of samples. If spin-flip occurs as electrons hopping from one electrode to another, the tunnel conductance (TC) and TMR are expected to be suppressed.

\footnotetext{
* Corresponding author.

E-mail address: liyun@ phys.ntu.edu.tw (Y. Li).
}

In the general case, the spin and orbital motions are not independent, i.e., a spin-orbit (SO) coupling exists, the crucial interaction which provides the spindependent potential. Conduction electrons lose memory of their spin orientation due to disorder scattering. So far, the theoretical treatment of disorder-assisted tunneling have not taken into account such spin-flip and spin-mixing mechanism as SO coupling. Experimentally, it has also been observed that the TMR depends critically on the interface condition [8]. The SO scattering due to interfacial roughness should definitely has a strong influence on the TC and TMR.

In this Letter, the effects of SO coupling at the FM/I(S) interface on the TC and TMR are investigated numerically within the tight-binding model. We extend the Landauer-Büttiker formalism to spin systems and apply it to a FM/I(S)/FM junction. The calcula- 
tions are performed using the recursive method for the real space Green's function. The spin-flip ratio and the spin relaxation due to the SO coupling are obtained. Zero temperature and zero bias limit are assumed for simplicity in this work.

The structure considered here consists of two semiinfinite FMs $\left(-\infty \prec i \preceq 0\right.$ and $\left.N_{x} \preceq i \prec \infty\right)$ separated by an insulator (semiconductor) layer ( $1 \preceq i \preceq$ $N_{x}-1$ ), where $N_{x}$ is the site along the $x$ (current) direction. Since it is physically transparent, the singleorbital tight-binding model is adopted to describe the simple cubic lattice with the lattice constant $a$ and with nearest-neighbor hopping, $t$, which is same for all pairs of nearest neighbors. The (100) axis is taking as the stacking direction. Along the cross-section a periodic structure with $N_{y} \times N_{z}$ supercell is considered, where $N_{y}$ and $N_{z}$ are the sites along the $y$ and $z$ axes, respectively.

The Hamiltonian is given by

$$
H=\frac{P^{2}}{2 m^{*}}+U(\mathbf{r})+\frac{\delta}{2} \vec{\mu}(\mathbf{r}) \cdot \vec{\sigma}+\lambda(\nabla U(\mathbf{r}) \times \vec{\sigma}) \cdot \hat{P},
$$

where the first and second terms are kinetic and potential energies, the third and forth represent exchange and spin-orbit interaction, respectively, $m^{*}$ is the effective mass of electron, $\delta$ the exchange splitting, $\vec{\mu}$ a unit vector in the direction of magnetization of FMs, $\vec{\sigma}$ the Pauli operator and $\hat{P}$ the momentum operator.

By discreting the spatial coordinate, the Hamiltonian is expressed as [9]

$$
\begin{aligned}
H= & \sum_{\mathbf{r}, \sigma, \sigma^{\prime}}\left(\varepsilon_{\mathbf{r}} \delta_{\sigma \sigma^{\prime}}+\frac{\delta_{\mathbf{r}}}{2} \vec{\mu}_{\mathbf{r}} \cdot \vec{\sigma}_{\sigma \sigma^{\prime}}\right) c_{\mathbf{r}, \sigma}^{+} c_{\mathbf{r}, \sigma^{\prime}} \\
& +t \sum_{\left\langle\mathbf{r}, \mathbf{r}^{\prime}\right\rangle \sigma} c_{\mathbf{r}, \sigma}^{+} c_{\mathbf{r}^{\prime}, \sigma}+H_{\mathrm{so}}, \\
H_{\mathrm{So}}= & i \alpha_{\mathrm{so}} \sum_{\mathbf{r}, \sigma, \sigma^{\prime}}\left[\Delta \varepsilon_{\mathbf{r} \pm \mathbf{a}_{\mathbf{y}}, \mathbf{r} \pm \mathbf{a}_{\mathbf{z}}} c_{\mathbf{r}, \sigma}^{+} c_{\mathbf{r} \pm \mathbf{a}_{\mathbf{y}} \pm \mathbf{a}_{\mathbf{z}}, \sigma^{\prime}} \sigma_{\sigma \sigma^{\prime}}^{x}\right. \\
& +\Delta \varepsilon_{\mathbf{r} \pm \mathbf{a}_{\mathbf{x}}, \mathbf{r} \pm \mathbf{a}_{\mathbf{z}}} c_{\mathbf{r}, \sigma}^{+} c_{\mathbf{r} \pm \mathbf{a}_{\mathbf{x}} \pm \mathbf{a}_{\mathbf{z}}, \sigma^{\prime}} \sigma_{\sigma \sigma^{\prime}}^{y} \\
& \left.+\Delta \varepsilon_{\mathbf{r} \pm \mathbf{a}_{\mathbf{x}}, \mathbf{r} \pm \mathbf{a}_{\mathbf{y}}} c_{\mathbf{r}, \sigma}^{+} c_{\mathbf{r} \pm \mathbf{a}_{\mathbf{x}} \pm \mathbf{a}_{\mathbf{y}}, \sigma^{\prime}} \sigma_{\sigma \sigma^{\prime}}^{z}\right], \\
t=- & \frac{\hbar^{2}}{2 m^{*} a^{2}}, \\
\alpha_{\mathrm{so}}= & \frac{\lambda \hbar}{a^{2}},
\end{aligned}
$$

where $c_{\mathbf{r}, \sigma^{+}}\left(c_{\mathbf{r}, \sigma^{\prime}}\right)$ is the creation (annihilation) operator of an electron with spin $\sigma\left(\sigma^{\prime}\right)$ at site $\mathbf{r}, \varepsilon_{\mathbf{r}}$ on-site energy and $\Delta \varepsilon_{\mathbf{r} \pm \mathbf{a}_{\mathbf{y}}, \mathbf{r} \pm \mathbf{a}_{\mathbf{z}}}=\varepsilon_{\mathbf{r} \pm \mathbf{a}_{\mathbf{y}}}-\varepsilon_{\mathbf{r} \pm \mathbf{a}_{\mathbf{z}}}, \mathbf{r} \pm \mathbf{a}_{\mathbf{y}}\left(\mathbf{a}_{\mathbf{z}}\right)$ denote the nearest neighbor sites from site $\mathbf{r}$ along $y$ (z) axis, $\mathbf{a}_{\mathbf{y}(\mathbf{z})}$ is the vector of length $a$ and parallel to the $y(z)$ axis, $\sigma_{\sigma \sigma^{\prime}}^{x}, \sigma_{\sigma \sigma^{\prime}}^{y}, \sigma_{\sigma \sigma^{\prime}}^{z}$ are Pauli matrix elements, $\alpha_{\text {so }}$ is spin-orbit parameter. The summation $\left\langle\mathbf{r}, \mathbf{r}^{\prime}\right\rangle$ runs over nearest neighbor sites. All energies are measured in unit of hopping integral $|t|$.

Extending the Landauer-Büttiker formalism to spin systems, the TC is expressed as $[9,10]$

$G_{\sigma \sigma^{\prime}}=\frac{e^{2}}{h} \operatorname{Tr}_{\mathbf{k}_{\|}}\left(\Gamma_{L}^{\sigma} G_{L R \sigma \sigma^{\prime}}^{+} \Gamma_{R}^{\sigma^{\prime}} G_{R L \sigma^{\prime} \sigma}^{-}\right)$,

where $\operatorname{Tr}$ denotes the trace for $2 N_{y} N_{z} \times 2 N_{y} N_{z}$ matrices. $\mathbf{k}_{\|}$is the wave vector in the transverse direction in the two-dimensional Brillouin zone corresponding to the supercell. $G^{+}$and $G^{-}$are retarded and advanced Green's function, respectively. $L$ and $R$ refer to the left and right $\mathrm{FM}$ electrodes, and

$$
\begin{aligned}
& \Gamma_{L}^{\sigma} \equiv i t^{2}\left[G_{L \sigma}^{+}(1)-G_{L \sigma}^{-}(1)\right], \\
& \Gamma_{R}^{\sigma^{\prime}} \equiv i t^{2}\left[G_{R \sigma^{\prime}}^{+}\left(N_{x}\right)-G_{R \sigma^{\prime}}^{-}\left(N_{x}\right)\right],
\end{aligned}
$$

where $G_{L \sigma}^{ \pm}(1)$ and $G_{R \sigma^{\prime}}^{ \pm}\left(N_{x}\right)$ are the surface Green's functions for the unconnected left and right electrodes. The interlayer Green's function $G_{L R \sigma \sigma^{\prime}}^{ \pm}$is calculated numerically using recursive method. Due to the SO coupling and magnetic impurity, the conductance is expressed as the sum of different spin channels, i.e.,

$G=G_{\mathrm{sc}}+G_{\mathrm{sf}}$,

$G_{\mathrm{sc}}=G_{\uparrow \uparrow}+G_{\downarrow \downarrow}$,

$G_{\text {sf }}=G_{\uparrow \downarrow}+G_{\downarrow \uparrow}$,

where $\uparrow \uparrow(\downarrow \downarrow)$ indicates that the magnetic moment of electron is parallel (anti-parallel) to the magnetization direction in the left and right FM leads, respectively. $G_{\text {sc }}\left(G_{\text {sf }}\right)$ is spin-conserving (spin-flip) term.

TMR ratio is defined as [11]

$\mathrm{TMR}=\frac{\Delta G}{\bar{G}}=\frac{G_{\mathrm{p}}-G_{\mathrm{ap}}}{\bar{G}}$,

where $G_{\mathrm{p}}\left(G_{\text {ap }}\right)$ is the TC for parallel (P) (anti-parallel (AP)) configuration of magnetization in FMs, $\bar{G}$ represents the average value of the two configurations.

A random substitutional disorder which is represented by randomness in the on-site energy at the $\mathrm{FM} / \mathrm{I}(\mathrm{S})$ interface in the right lead is considered (similar results may be expected for the case with two 
disordered regions in the left and right interfaces, respectively). The disorder-induced SO coupling is a random-site potential which induces momentum scattering accompanied by spin-flip process. The ratio $\tau_{\mathrm{so}} / \tau_{\mathrm{m}}$ is $[9]$

$\frac{\tau_{\mathrm{so}}}{\tau_{\mathrm{m}}}=\left(2 \sqrt{2} \frac{E_{\mathrm{F}}}{|t|} \alpha_{\mathrm{so}}\right)^{-2}$,

where $\tau_{\mathrm{so}}\left(\tau_{\mathrm{m}}\right)$ is spin (momentum) relaxation time, and therefore the spin-relaxation length [9] is

$l_{\mathrm{so}}=l_{\mathrm{m}} \sqrt{\frac{\tau_{\mathrm{so}}}{\tau_{\mathrm{m}}}}=\frac{\pi a|t|^{3}}{\sqrt{2} E_{\mathrm{F}} \alpha_{\mathrm{so}}\left\langle\left(\varepsilon_{\mathbf{r}}-\bar{\varepsilon}\right)^{2}\right\rangle_{\mathrm{c}}}$

for diffusive transport, i.e., $\tau_{\mathrm{so}} \gg \tau_{\mathrm{m}}$, where $l_{\mathrm{m}}$ is the mean-free path. When $\tau_{\text {so }}$ is of the same order or shorter than $\tau_{\mathrm{m}}$, that is, if $\tau_{\mathrm{so}} \sim \tau_{\mathrm{m}}$, then [9]

$l_{\mathrm{so}}=l_{\mathrm{m}} \frac{\tau_{\mathrm{so}}}{\tau_{\mathrm{m}}}=\frac{\pi a|t|^{4}}{\left(2 E_{\mathrm{F}} \alpha_{\mathrm{so}}\right)^{2}\left\langle\left(\varepsilon_{\mathbf{r}}-\bar{\varepsilon}\right)^{2}\right\rangle_{\mathrm{c}}}$

for ballistic transport, where $\langle\cdots\rangle_{\mathrm{c}}$ represents the configuration average, $E_{\mathrm{F}}$ is measured from the bottom of the band.

For the numerical calculation, the hopping integral $t$ is taken to be -1 . The spin independent on-site energy $\varepsilon_{\mathbf{r}}$ is set to be 0 for the FMs except the disorder sites. Inside the barrier $(1 \preceq i \preceq 4), \varepsilon_{\mathbf{r}}$ is chosen to be the barrier height $2|t|$. In the disordered region, the concentrations of magnetic impurities with on-site energy $\varepsilon_{i}$ and $-\varepsilon_{i}$ are taken to be equal. The size of the transverse cross section is $10 \times 10$, the Fermi energy and the exchange splitting are taken to be $0.9|t|$ and $1.0|t|[12]$, respectively.

The spin-flip ratio, $G_{\mathrm{sf}} / G$, as a function of the disordered layer (DL) thickness at the interface (the thickness of barrier is 4 monolayers (MLs)) for $\alpha_{\mathrm{so}}=$ 0.15 is shown in Fig. 1. It increases linearly with different slope for $\mathrm{P}$ and AP alignment of magnetization in the two FMs. From our calculation, the spin-flip ratio increases by a factor of 2 and of 4 for $\mathrm{P}$ and AP configurations, respectively. In spin-conserving tunneling process, conductance is larger when the magnetizations of the two FMs are parallel than when they are antiparallel, which arises from the difference between the densities of majority and minority spin electrons at the Fermi level in the two FM electrodes. In contrast, the spin-flip (SF) scattering is much stronger for AP configuration of the magnetization in the two FM electrodes due to the reversal of the spin dependence

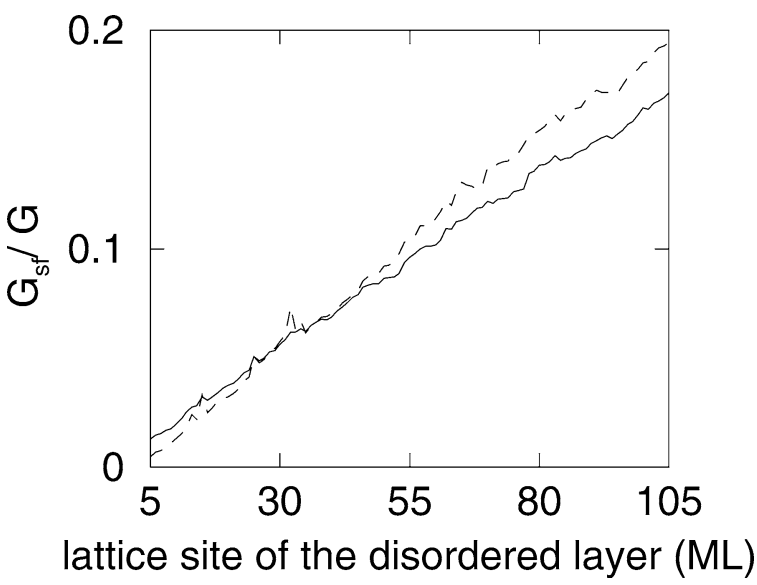

Fig. 1. The spin-flip ratio, $G_{\mathrm{sf}} / G$, as a function of disordered layer at the interface for $\mathrm{P}$ (solid line) and $\mathrm{AP}$ (dashed line) configurations, respectively, for $\alpha_{\mathrm{So}}=0.15$. The thickness of barrier is taken to be 4 MLs. The parameters used are $E_{\mathrm{F}}=0.9|t|, \delta=1.0|t|, U=2.0|t|$, $\epsilon_{1}=0.3|t|, \epsilon_{2}=-0.3|t|, c_{1}=0.5$.

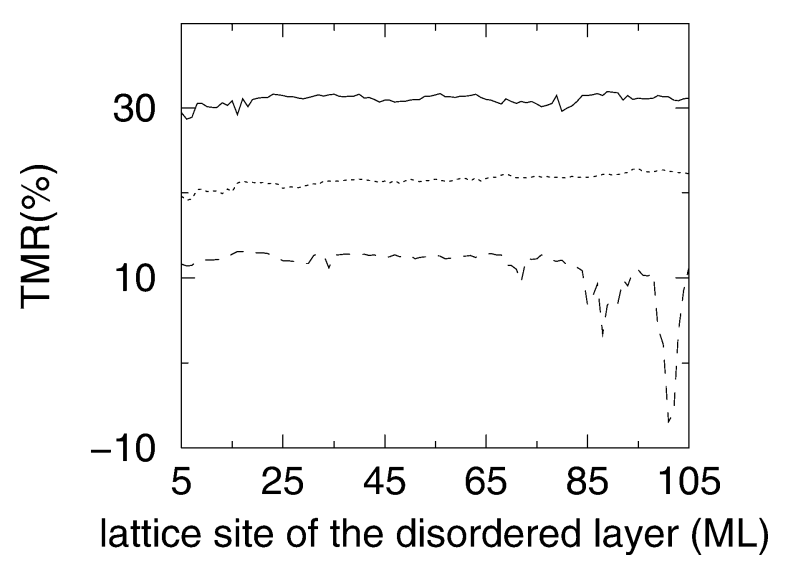

Fig. 2. The variation of TMR with the disordered layer with and without SO scattering, respectively. The parameters used are the same as in Fig. 1. $\alpha_{\text {so }}$ : 0 (solid line); 0.15 (dotted line); 0.2 (dashed line).

of final density of state (DOS). When a spin-flip event occurs, a spin-up electron tunneling from left FM electrode enter a spin-down empty state in the right FM lead, and vice versa. It seems as if the magnetization of the right FM has been reversed.

The spin-flip process due to SO coupling causes a significant reduction of TMR. Fig. 2 shows the variation of TMR with the DL layer at the interface. It decreases by $65 \%$ due to SO scattering for $\alpha_{\text {so }}=0.15$. 
The spin-conserving tunneling gives the usual negative TMR, whereas due to the reversal of the role of up and down spin final DOSs for electrons undergoing SF scattering, the spin-flip process contribute to an inverse magnetoresistance (MR) (see Eq. (9)). Thus the total TMR, which is the sum of the spin-conserving (negative) and spin-flip (positive) contributions is reduced. Furthermore we find that the surface spin scattering predominates which suggests that the interfacial states play a key role in the tunneling process (see Fig. 2). The feature of the reduction of TMR changes with the type and degree of disorder and the SO coupling strength. The SO coupling can remove the spin degeneracy in the band structure, which leads to the splitting of the conduction band. Resonant tunneling occurs which can be ascribed to the combined spin resonance $[13,14]$ of electrons corresponding to the hopping between the SO-split energy levels. Such phenomenon has been observed experimentally [15-18]. The ratio $\tau_{\mathrm{so}} / \tau_{\mathrm{m}}$ is approximately 4 and the spin relaxation length, $l_{\text {so }}$ due to SO coupling is about $200 \mathrm{MLs}$ for $\alpha_{\mathrm{so}}=0.2$ for the quasi-ballistic transport process considered here.

In conclusion, the effects of interfacial roughness on the TC and TMR are dramatic. The spin-flip process due to SO coupling contributes to an inverse (positive) MR. The reason is the reversal of the spin dependence of the final DOSs in such process, i.e., the role of up and down spin final DOSs is reversed for electrons undergoing SF scattering. Resonant tunneling appears due to the hopping between the SO-split energy levels. Since the SF scattering is much stronger for the AP configuration of the magnetization in the two FM electrodes, it can be expected that the total TMR will be inversed (positive) when the SF scattering is larger than some critical point. The effects of bias voltage on the TC and TMR are under investigation, our preliminary results show the TMR decreases nonlinearly with the bias voltage, more details need to be studied.

\section{Acknowledgements}

Y.L. thanks Max-Planck-Institut für Mikrostrukturphysik for the financial support during her stay.

\section{References}

[1] T. Miyazaki, N. Tezuka, J. Magn. Magn. Mater. 139 (1995) L231.

[2] J.S. Moodera, L. Kinder, T. Wong, R. Meservey, Phys. Rev. Lett. 74 (1995) 3273.

[3] T. Miyazaki, N. Tezuka, J. Appl. Phys. 79 (1996) 6262

[4] J. Moodera, L. Kinder, J. Appl. Phys. 79 (1996) 4724.

[5] S. Zhang, P.M. Levy, A.C. Marley, S.S.P. Parkin, Phys. Rev. Lett. 79 (1997) 3744.

[6] J. Moodera et al., Phys. Rev. Lett. 80 (1998) 2941.

[7] S. Gider et al., Science 281 (1998) 797.

[8] J.M. Teresa, A. Barthelemy, A. Fert et al., Science 286 (1999) 507.

[9] Y. Li, unpublished.

[10] S. Datta, J. Phys. Condens. Matter. 2 (1990) 8023.

[11] M. Julliere, Phys. Lett. A 54 (1975) 225.

[12] J. Mathon, Phys. Rev. B 56 (1997) 11810.

[13] E.I. Rashba, Fiz. Tverd. Tela (Leningrad) 2 (1960) 1224, Sov. Phys. Solid State 2 (1960) 1109.

[14] E.I. Rashba, Usp. Fiz. Nauk 84 (1964) 557, Sov. Phys. Usp. 7 (1965) 82.

[15] S.J. Bending, M.R. Beasley, Phys. Rev. Lett. 55 (1985) 324.

[16] M. Naito, M.R. Beasley, Phys. Rev. B 35 (1987) 2548.

[17] R. Meservey, P.M. Tedrow, J.S. Brooks, J. Appl. Phys. 53 (1982) 1563.

[18] G.A. Gibson, R. Meservey, J. Appl. Phys. 58 (1985) 1584. 